\title{
Laser measurement of torsional vibrations/longitudinal creepage of a railway wheel set on a scaled test bench
}

\author{
C. Collette *, A. Preumont \\ Mechanical Engineering and Robotics Department, University of Brussels, 50 av. F.D. Roosevelt, 1050 Brussels, Belgium
}

\section{A R T I C L E I N F O}

Available online 5 May 2008

Keywords:

Torsional vibrations

Scaled roller rig

Multi-body model

Laser measurement

\begin{abstract}
A B S T R A C T
Since more than one century, test benches remain an essential tool to study various aspects of the railway dynamics such as for instance running stability, safety or even ride comfort. For each of these applications, the knowledge of the contact conditions (forces and relative displacements) between the wheel and the rail is a necessary condition to develop a sound understanding of the physical phenomena. More specifically, as soon as the longitudinal dynamics of the vehicle-track system is involved in the study (like for the performance of a locomotive, the rolling noise or rail corrugation), a precise measure of the longitudinal creepage between the wheel and the rail is needed to verify numerical predictions from theoretical models. In this paper, we focus on the measurement of torsional vibrations of a scaled wheel set which is rolling on a roller (representing infinite rails). First, a theoretical overview of the conditions under which these torsional vibrations are excited is given. Then, the experimental set-up used to study the phenomenon is presented. During the experiment, the wheel set torsional vibrations are measured using the rotational laser Doppler vibrometer, and the measure is used to calculate the longitudinal creepage of the wheel. Results are compared with outputs of a multibody model of the test bench.
\end{abstract}

(c) 2008 Elsevier Ltd. All rights reserved.

\section{Introduction}

Under specific operating conditions, wheel-rail creep forces excite torsional vibrations of metro wheel sets, which are responsible for a roll-slip phenomenon between the wheel and the rail. Depending on circumstances, these oscillations correspond either to a resonance frequency of the wheel set or to another frequency. In both cases these vibrations are prone to develop a wavy wear of the railhead surface, known as rail rutting corrugation [1]. In a recent literature review, it has been mentioned that '...there are good grounds to believe that rail rutting corrugation is the principal wavelength fixing mechanism for corrugation in general on metro railways [2]'. Different types of solutions exist to mitigate this type of wear, for instance: improvement of the steering capabilities of the bogie [1], friction modifiers [3], decrease the longitudinal creep forces. In this paper, an alternative solution is investigated, consisting of a dynamic vibration absorber (DVA) tuned to a torsional resonance of the wheel set. This solution was originally suggested in [4], but had not been tested yet. The efficiency of the DVA is evaluated

\footnotetext{
* Corresponding author. Tel.: +3226504662; fax: +3226504660.

E-mail address: christophe.collette@ulb.ac.be (C. Collette).

URL: http://www.ulb.ac.be/scmero (C. Collette).
}

experimentally by measuring the torsional vibrations of the wheel set using the vibrometer.

The paper is organized as follows. Section 2 describes the basic conditions to be fulfilled for rutting corrugation to appear on the rail surface. In Section 3, an experiment reproducing typical operating conditions in which rutting corrugation appears is considered. The experimental set-up is a quarter-scale test bench from the New Technologies Laboratory (INRETS-France) [5]. Section 4 presents briefly the working principle of the rotational laser Doppler vibrometer and Section 5 presents a multi-body model of the scaled test bench. In Section 6, the numerical efficiency of the DVA in mitigating rail rutting corrugation is evaluated in terms of the frictional power dissipated in the contact patch. Measurements are additionally compared with outputs from the multi-body model of the test bench which is portraying the experiment.

\section{Rail rutting corrugation}

\subsection{Background}

In the seventies [6], it was proved experimentally that the contact stiffness is involved in the corrugation formation on a two-disc test bench. The theoretical foundations for a detailed 


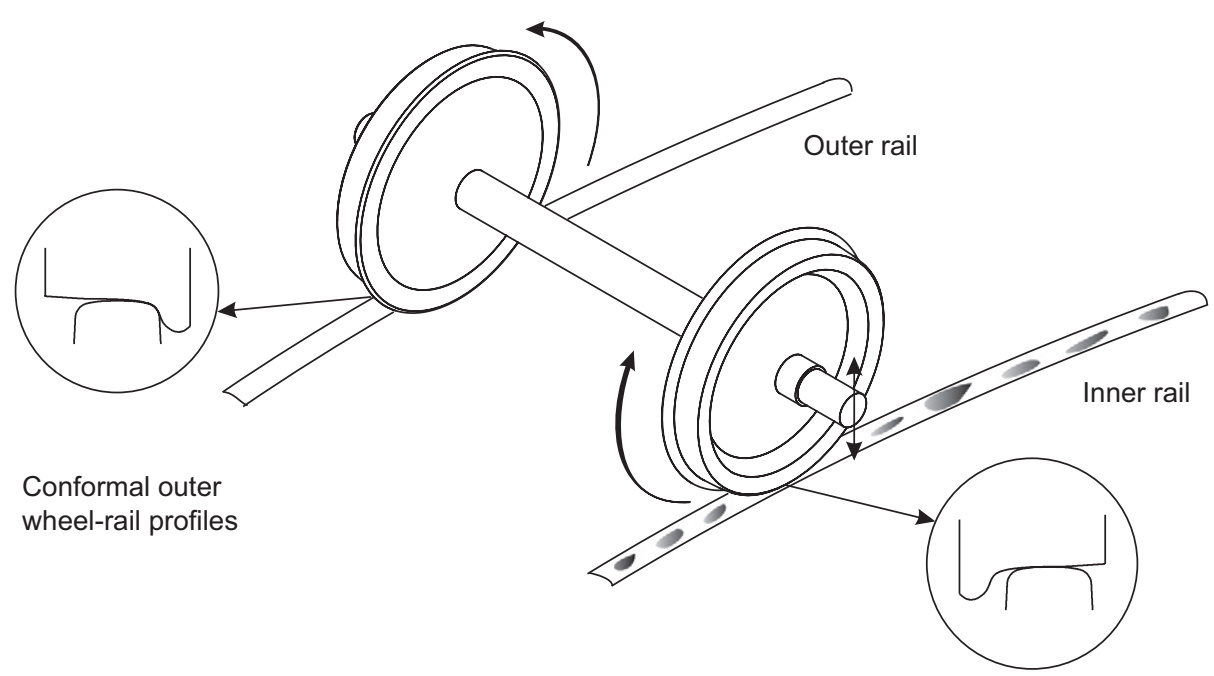

Fig. 1. Rutting corrugation; corrugation appears most of the time on the low (inner) rail because of the lower axle load and non-conformal contact.

physical explanation of these experimental observations were presented in [7], as well as conjectures on the specific mechanism involved in the formation of corrugations. The mode of vibration was termed the 'contact mode'. The mathematical model developed at that time was however too simple as only the vertical dynamics were taken into account. A more complete mathematical model was developed in [8] to describe rail corrugation as a wear process resulting from the combination of torsional vibrations of the drive wheels and longitudinal vibrations of the rail. Using their simple model, they were additionally able to derive an analytical formulation of the frictional power to characterize longitudinal shape of rail corrugation. A model in which torsional vibrations of the wheel set are the main causes for rail corrugation to develop was presented in [9]. This type of corrugation was definitively classified as rail 'rutting corrugation' in [1]. More recently, a formation mechanism of rail corrugation involving torsional vibrations of the wheel set has been proposed in [10]. In this case, torsional oscillations are only responsible for the creep process, and sustained by the vertical vibrations of the unsprung mass on the contact stiffness. This wavelength fixing mechanism is explained in more details in the next section.

\subsection{Wavelength fixing mechanism}

Wheel and rail profiles impose the railway vehicle to follow the track. This constrained motion induces high wheel-rail contact creep forces, and stresses in the vehicle-track system. The type of stress depends on the geometry of the system and the operating conditions. In this paper, only torsional stresses induced in the wheel set are considered. Among the phenomena responsible for this type of stresses, one can cite: the difference in rail length of curved track sections, the wear of wheel and rail profiles, a bad adaptation of the vehicle speed to the track superelevation and driving torques applied on the gear box. Above the sticking limit (defined as the product of the friction coefficient and the wheel load), additional creep forces impose a constant slip between the two bodies in contact. During the passage of the vehicle, the surface roughness impose a relative displacement between the wheel and the rail at each contact point, which is exciting the vertical dynamic contact forces. High oscillations of the vertical contact force impose the relaxation of the stress accumulated in the wheel set at the same frequency. It results in a 'roll-slip' motion of the wheel at the frequencies of these resonances. For a given roughness spectrum, the vertical force spectrum is determined by the dynamic characteristics of the vehicle-track system. From a practical point of view, high variations of the vertical force between the wheel and the rail correspond to either (i) a bouncing of the unsprung mass of the vehicle on the support stiffness (the corresponding wavelength fixing mechanism is explained in $[10,11]$ ) or (ii) a resonance already existing in the direct receptance of the track alone, for instance a resonance of the sleeper.

Even if the torsional resonance is not a necessary condition for this wavelength fixing mechanism to appear, the longitudinal creepage between the wheel and the rail will of course increase when the frequency of a torsional resonance of the wheel set approximately equals a resonance frequency of the rail direct receptance. A drawing of the mechanism is shown in Fig. 1; corrugation usually appears on the rail where the dynamic load is the lowest. For instance in the case of a curved track, corrugation appears usually on the low (inner) rail like depicted in Fig. 1.

\section{Experimental set-up}

The test bench considered in the experiment (Fig. 2) is a quarter-scale roller rig, constituted of a scaled wheel set which is rolling on a roller representing infinite rails. The wheel set is attached to a half-bogie through geometrically scaled primary suspensions. The roller is driven by an electric motor (actuator 6) through a transmission belt. On this bench, the lateral displacement and the yaw angle of the wheel set can be fixed independently using adjusting devices 7 and 8, respectively. Longitudinal and lateral contact forces are measured with DC force sensors 12, 13 and 11, respectively (Fig. 2(b)). The half-bogie load is measured with a sensor 5 and the variable vertical contact force is measured using a piezoelectric force transducer connected to the shaker (sensor 15). The rotational speed is measured using a tachometer (sensor 10) and the torsional vibrations are measured using a rotational laser Doppler vibrometer (sensor 16). Torsional vibrations can also, in principle, be measured by the tachometer, but it turns out that the signal is more noisy at high frequency. Additionally, because of high vibration amplitudes imposed by the shaker, better results have been obtained when the laser beam points to the wheel where no load variation is applied.

The wavelength fixing mechanism described in Section 2.2 is reproduced as follows. In the curve, the outer rail is longer than the inner one. Due to the poor steering capabilities of the front 


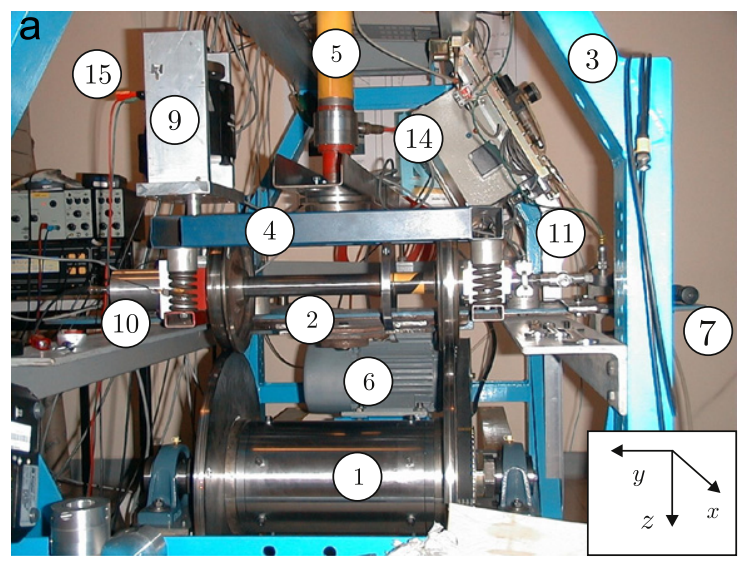

Test bench structure:

1. Roller

2. Wheel set

3. Metallic structure

4. Half bogie

Actuators:

5. Car body load

6. Driving motor

7. Lateral displacement

of the wheel set

8. Yaw angle of the wheel set

9. Shaker

Sensors:

10. Tachometer

11. Lateral force

12. Longitudinal force (right)

13. Longitudinal force (left)

14. Vertical force

b
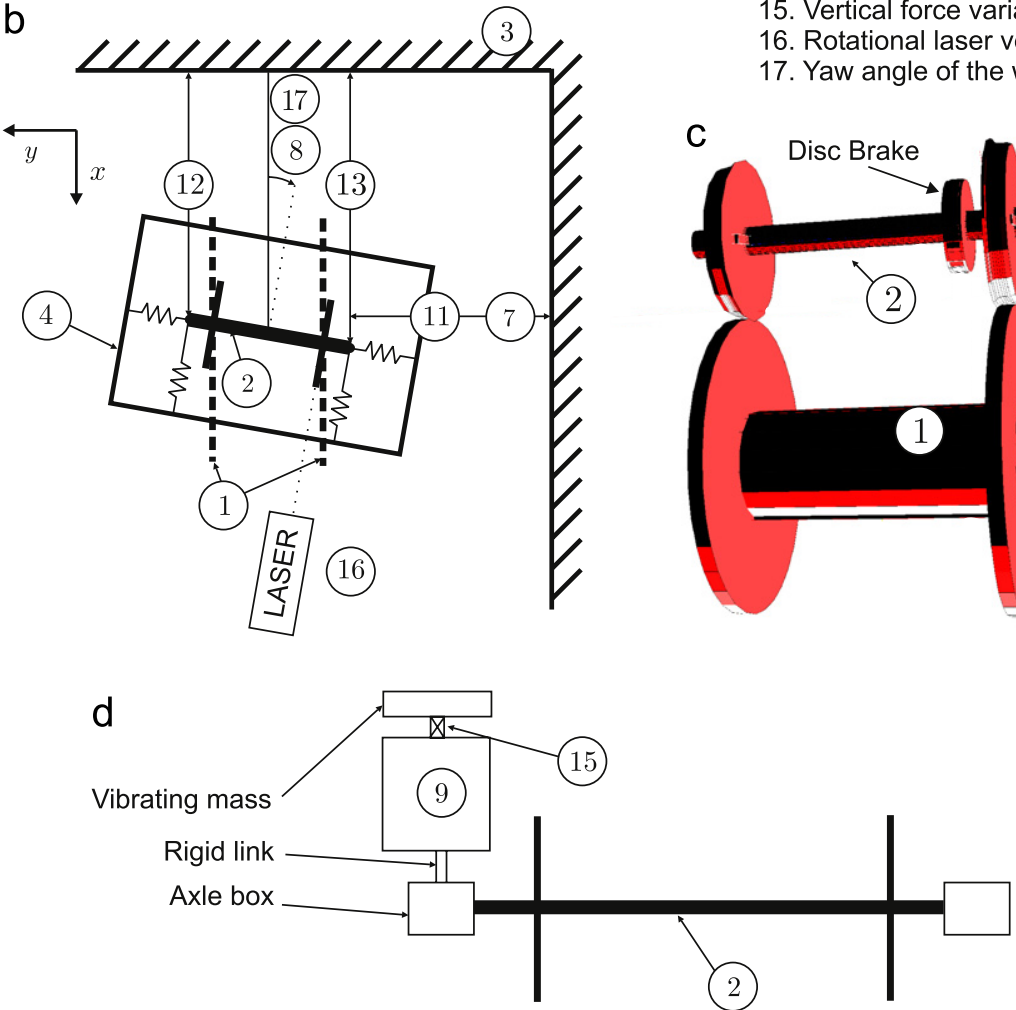

.

16. Rotational laser velocimeter

17. Yaw angle of the wheel set

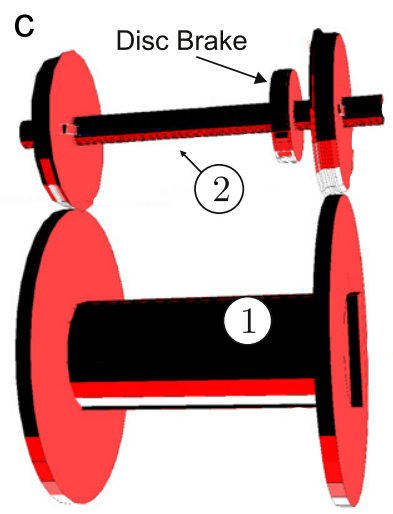

Fig. 2. (a) Picture of the $1 / 4$ scaled test bench; (b) simplified drawing of a top view of the bench; (c) multi-body model of the test bench; (d) simplified front view.

bogie in curve, the difference in rolling radii is insufficient to compensate the difference of rail length. As a consequence, a torsion is induced in the wheel set. On the bench, as both rails have the same length, the difference in rolling radii is imposed by a lateral displacement applied to the wheel set, resulting in a torsion of the wheel set due to the asymmetry of the wheel profiles.

Then, vertical force variations impose the wheel set to be twisted periodically and roll-slip oscillations between the rail and the wheel occur, creating a wavy wear on the surface of the inner rail. However, on the test bench, as no clear vertical resonance is visible in the direct receptance of the roller in the frequency range of the torsional resonance, the rail roughness is not sufficient to generate by itself any normal force variations high enough for rail corrugation to develop (see Section 2.2). For this reason, the vertical load variations are imposed by a shaker fixed directly on the inner axle box (actuator 9, Fig. 2(d)), simulating the vertical force variations imposed by the rail roughness to the wheel set.
Although the periodicity of the oscillations (i.e. the wavelength of rail corrugation) is indeed fixed at the frequency of the vertical load variations [12,11], the phenomenon will be maximum when this frequency is close to a torsional resonance of the wheel set. To investigate the impact of a vertical excitation at other frequencies than the resonance of the wheel set on wear, a force with a sweep sine frequency is applied by the shaker. The contact friction variations are evaluated from the measurements of the longitudinal creepage.

The experiment to reproduce the mechanism of rutting corrugation on the scaled bench has been performed in the following conditions:

- wheel set linear speed: $V=5 \mathrm{~m} / \mathrm{s}$;

- lateral force: $F_{y}=700 \mathrm{~N}$;

- vertical excitation frequency: $\omega_{\text {sweep }} \in[170,250] \mathrm{Hz}$ (Duration: $20 \mathrm{~s})$

- vertical excitation force: $\Delta N=40 \mathrm{~N}$. 


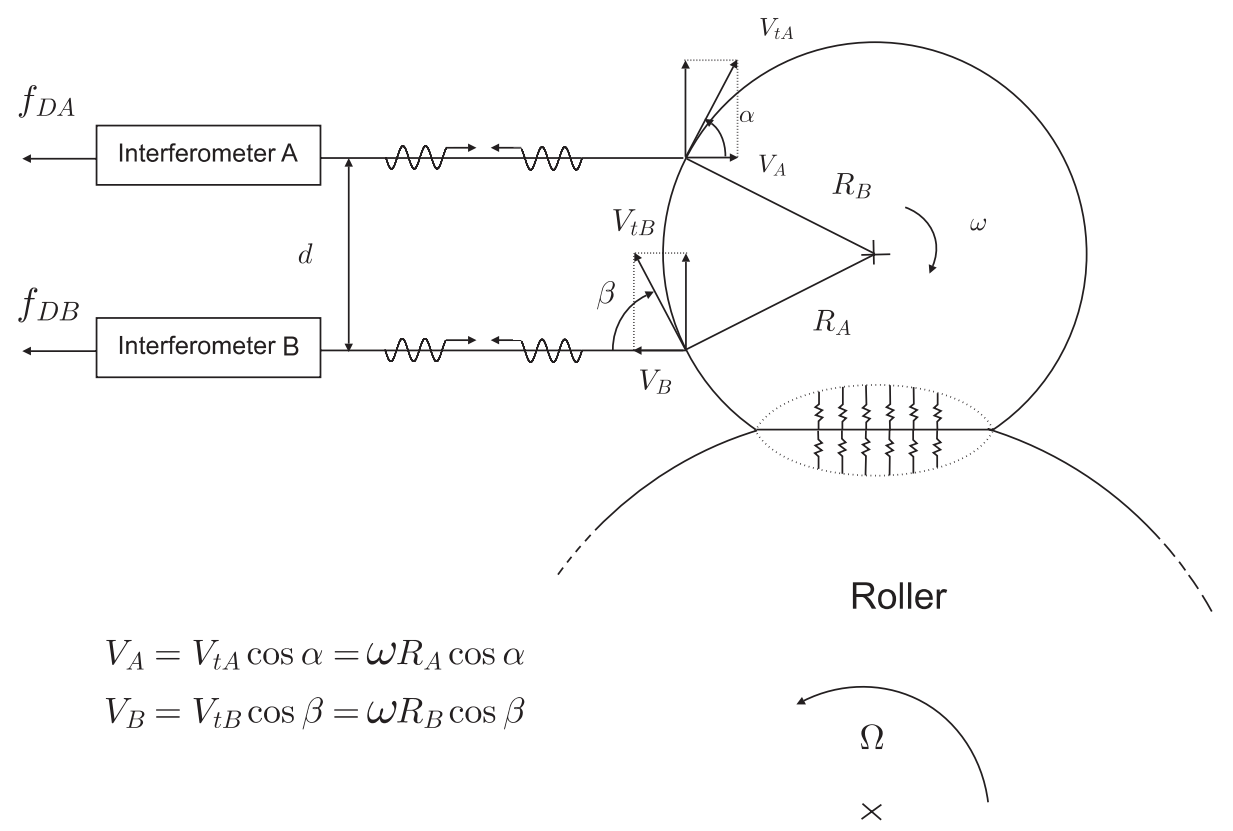

Fig. 3. Acquisition of the angular velocity using the two-point measurement [14].

\section{Working principle of the rotational laser vibrometer}

Each point on the circumference of a rotating part, with an angular velocity $\omega$ (Fig. 3) has a tangential velocity which is dependent on the rotational radius $R$. This translational velocity is the vectorial sum of two components: one in the direction of the incident laser beam ( $V_{\mathrm{A}}$ and $V_{\mathrm{B}}$ in Fig. 3) and another perpendicular. The rotational laser Doppler vibrometer determine the angular velocity $\omega$ as follows. The measuring arrangement consists of two interferometers from which the laser beams are positioned parallel to each other at a distance $d$ (Fig. 3). Each interferometer acquires a translational velocity $V_{\mathrm{A}}$ and $V_{\mathrm{B}}$. From the back scattered beams, each of the interferometer produces a Doppler frequency $f_{\mathrm{DA}}$ and $f_{\mathrm{DB}}$, related to the translational speeds of the measurement points $\mathrm{A}$ and $\mathrm{B}$ with the following formulas:

$f_{\mathrm{DA}}=2 V_{\mathrm{A}} / \lambda=2 \omega R_{\mathrm{A}} \cos \alpha / \lambda$;

$f_{\mathrm{DB}}=2 V_{\mathrm{B}} / \lambda=2 \omega R_{\mathrm{B}} \cos \beta / \lambda$

where $\lambda=633 \mathrm{~nm}$ is the wavelength of the HeNe laser and $\alpha, \beta, R_{\mathrm{A}}$, $R_{\mathrm{B}}$ are geometrical parameters defined in Fig. 3. Additionally, the geometrical relationship between $\alpha, \beta$ and $d$ is

$d=R_{\mathrm{A}} \cos \alpha+R_{\mathrm{B}} \cos \beta$

Using Eq. (2), we get from Fig. 3:

$V_{\mathrm{A}}+V_{\mathrm{B}}=\omega(r \cos \alpha+r \cos \beta)=\omega d$

and accordingly the angular frequency $\omega$. On the scaled test bench, the longitudinal creepage is written as

$v_{x}=\frac{V_{\text {Roller }}-V_{\text {Wheel set }}}{V_{\text {Roller }}}=\frac{\Omega R-\omega r}{\Omega R}$

where $r=\left(R_{\mathrm{A}}+R_{\mathrm{B}}\right) / 2$ is the average radius of the wheel.

\section{Multi-body model}

The multi-body model of the test bench (Fig. 2(c)) has been developed in SIMPACK. It is constituted of a flexible wheel set which is rolling on a rigid body roller, rolling at a constant linear speed of $5 \mathrm{~m} / \mathrm{s}$. The wheel set is represented by a finite element model including its first six modes (two torsional modes and four bending modes), and is linked to the inertial frame through the primary suspensions. The lateral force of $F_{y}=700 \mathrm{~N}$ is imposed by shifting the lateral coordinate of the attaching point of the primary suspension on the inertial frame of $9 \mathrm{~mm}$. The sweep sine force is applied on the axle box corresponding to the nonconformal contact like depicted in Fig. 1.

\section{Experimental results}

The DVA used for the simulation is a rotational inertia, fixed onto the wheel set axle through a spring in parallel with a dashpot. The parameters of the DVA have been chosen using the so-called equal peak method [13]. The mechanical realization of the DVA is an iron ring, fixed onto the wheel set axle through a layer of viscoelastic material. The simulated and measured power spectral densities of the rotational speed of the wheel without and with the DVA are compared in Figs. 4(a) and (b), respectively. In each case, the DVA has been found to reduce the peak amplitude of the power spectral density by a factor 3 . The measured spectral densities have been averaged on three tests with and without the DVA.

\section{Conclusions}

In this paper, it has been shown that torsional vibrations of a scaled wheel set can be measured using a rotational laser Doppler vibrometer in operating conditions, reproducing the wavelength fixing mechanism of rutting corrugation. Experimental results have been shown to correlate to outputs of a multi-body model which is portraying the experiments. Additionally, it has been shown experimentally that, close to the torsional resonance of the wheel set, a passive dynamic vibration absorber can reduce the amplitude of these torsional vibrations by a factor 3 , which is again consistent with numerical calculations. 
a

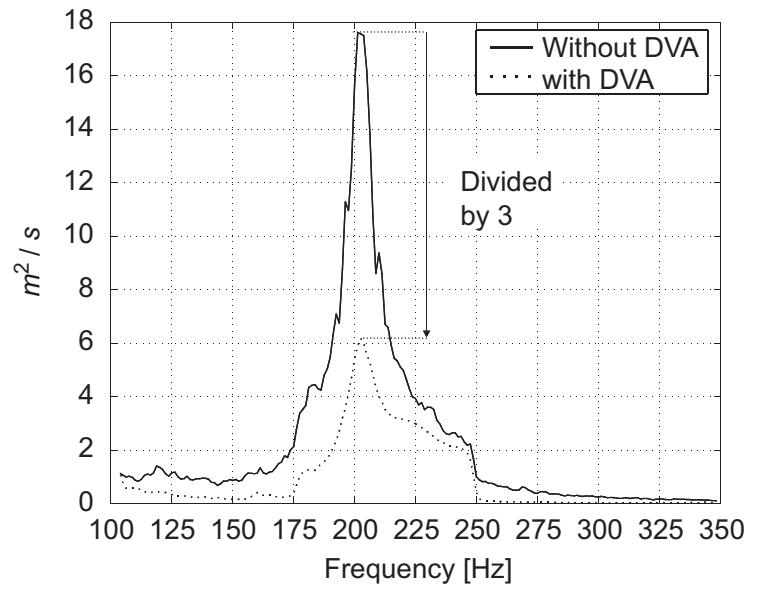

b

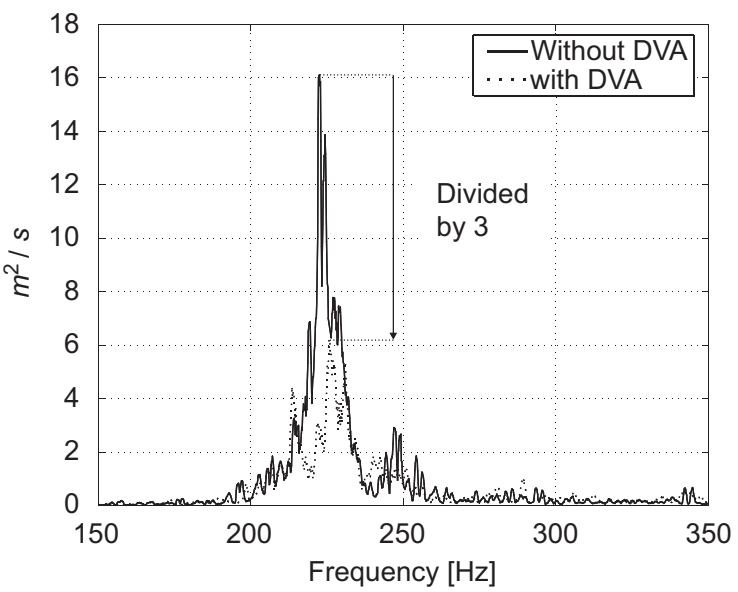

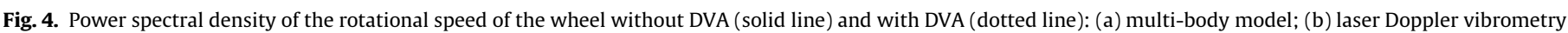
measurements.

\section{References}

[1] Grassie SL, Kalousek J. Rail corrugation: characteristics, causes and treatments. J Rail Rapid Transit 1993;207:57-68.

[2] Grassie SL. Rail corrugation: advances in measurement, understanding and treatment. Wear 2005;258:1224-34.

[3] Eadie DT, Kalousek J, Chiddick KC. The role of high positive friction (HPF) modifier in the control of short pitch corrugations and related phenomena. Wear 2002;253:185-92.

[4] Transit Cooperative Research Program. Rail corrugation mitigation in transit, June 1998.

[5] Chollet H. Etude En Similitude Mécanique Des Efforts Tangents Au Contact Roue-Rail. PhD thesis, Université Paris 6; 1991.

[6] Carson MR, Johnson KL. Surface corrugations, spontaneously generated in a rolling contact disc machine. Wear 1971;17:59-72.

[7] Ranganath Nayak P. Contact vibrations. J Sound Vib 1972;22:297-322.
[8] Brockley CA, Ko PL. An investigation of rail corrugation using friction-induced vibration theory. Wear 1988;128:99-106.

[9] Hayes WF, Tucker HG. Wheel set-track resonance as a possible source of corrugation wear. Wear 1991;144:211-26.

[10] Matsumoto A, Sato Y, Tanimoto M, Kang Q. Study on the formation mechanism of rail corrugation on curved track. Vehicle Syst Dyn Suppl 1996;25:450-65.

[11] Matsumoto A, Sato Y, Hiroyuki O, Tanimoto M, Oka Y, Miyauchi E. Formation mechanism and countermeasures of rail corrugation on curved track. Wear 2002;253:178-84.

[12] Matsumoto A, Sato Y, Nakata M, Tanimoto M, Qi K. Wheel-rail contact mechanics at full scale on the test stand. Wear 1996;191:101-6.

[13] Ormondroyd J, Den Hartog JP. The theory of the damped vibration absorber. Trans Am Soc Mech Eng 1928;50:7.

[14] Polytec. Rotational vibrometer OFV-400 (user manual); 2005. 